\title{
Community-Based User Experience: Evaluating the Usability of Health Insurance Information With Immigrant Patients
}

Emma J. Rose

University of Washington Tacoma, ejrose@uw.edu

R. Racadio

K. Wong

S. Nguyen

J. Kim

See next page for additional authors

Follow this and additional works at: https://digitalcommons.tacoma.uw.edu/ias_pub

\section{Recommended Citation}

Rose, Emma J.; Racadio, R.; Wong, K.; Nguyen, S.; Kim, J.; and Zahler, A., "Community-Based User Experience: Evaluating the Usability of Health Insurance Information With Immigrant Patients" (2017). SIAS Faculty Publications. 756.

https://digitalcommons.tacoma.uw.edu/ias_pub/756 
Authors

Emma J. Rose, R. Racadio, K. Wong, S. Nguyen, J. Kim, and A. Zahler 


\section{Community-Based User Experience:}

\section{Evaluating the Usability of Health Insurance Information with Immigrant Patients}

-Emma J. Rose, Robert Racadio, Kalen Wong, Shally Nguyen, JeE Kim, and Abbie

ZAHLER

Manuscript received April 13, 2016; initial decision May 23, 2016; revised October 13, 2016;

accepted October 14, 2016. Date of current version: November 25, 2016. (Corresponding author:

Emma J. Rose.)

Emma J. Rose is with the University of Washington Tacoma, Tacoma, WA 98402 USA [email: ejrose@uw.edu].

Robert Racadio is with the University of Washington Seattle, Seattle, WA 98195 USA [email: racadio@uw.edu ].

Kalen Wong is with the University of Washington Seattle, Seattle, WA 98195 USA [email:

yellowcurry@gmail.com].

Shally Nguyen is with the University of Washington Seattle, Seattle, WA 98195 USA [email: shally.ntmh@gmail.com].

Jee Kim is with SEIU 775 Benefits Group, Seattle, WA 98104 USA [email:

jekim0405@gmail.com].

Abbie Zahler is with Foundation for Healthy Generations, International Community Health

Services, Seattle, WA 98119 USA [email: abbiez@healthygen.org]. 
Abstract-User experience $(U X)$, a common practice in corporate settings, is new for many nonprofits organizations. This case study details a community-based research project between nonprofit staff at a community health center and UX professionals to improve the design and usability of a document designed to help immigrant patients sign up for health insurance. UX professionals may need to adapt and be flexible with their efforts, but can offer valuable skills to community partners. Research questions: 1. What are the information needs and barriers faced by immigrant populations signing up for health insurance? 2. How does a usability study, adapted to meet the needs of immigrant populations, inform the design of a supplemental guidebook about health insurance? 3. What are the challenges and opportunities when engaging in community-based UX research projects? Situating the case: Other community-based research projects in technical communication and UX point to the need for a clear conceptualization of participation, a strong partnership with nonprofits, and the need to develop meaningful and actionable insights. Further, when conducting studies with immigrant populations, the role of the translator on the research team is crucial. Methodology: As a community-based research project focused on the collaborative generation of practical knowledge, we conducted a usability study with 12 participants in two language groups, Chinese and Vietnamese, to evaluate the design and usability of a guidebook designed to provide guidance about enrolling in a health insurance plan. Data were analyzed to identify usability concerns and used to inform a second iteration of the guidebook. About the case: Immigrant populations struggle to sign up for health insurance for a variety of reasons, including limited English and health insurance literacy. As a result, a nonprofit community health center developed a guidebook to support immigrant populations. Version 1 of this guidebook was evaluated in a usability study, with results showing that users 
struggled to correctly choose a plan, determine their eligibility, and interpret abstract examples. As a result, Version 2 was designed to support the in-person experience, reduce visual complexity, and support patients' key questions. Conclusions: Community-based UX collaborations can amplify the expertise of UX and nonprofit professionals. However, UX methods may need to be adapted in community-based projects to better incorporate local knowledge and needs.

Index Terms - user experience, usability, health insurance, design, immigrant populations

\section{INTRODUCTION}

Over the past 30 years, organizations have shifted their approach in designing information and communication technologies from a system-centered approach to a user-centered approach [1]. However, this shift has not been experienced evenly across all types of organizations. Non-profit organizations may be newer to the practice of user experience (UX) [2] and face particular constraints and challenges, including working with limited resources and designing information and services for vulnerable populations.

UX practices such as audience and task analysis, technical writing, and usability testing can help nonprofit organizations pursue their missions. UX professionals are well positioned to partner with nonprofit organizations to collaborate on UX projects. By engaging with nonprofits, UX professionals can deepen their own practice by engaging in creative problem solving within settings with constrained resources and working with diverse target users and audiences. 
This paper presents a case study of a community-based UX design project at a community health center, a nonprofit organization providing care to medically underserved populations. This case study focuses on two components:

- The design and usability study of a guidebook that was created to help patients sign up for health insurance

- The collaboration between the nonprofit staff and the UX professionals external to the organization

The following research questions drove this case:

RQ1. What are the information needs and barriers faced by immigrant populations signing up for health insurance?

RQ2. How does a usability study, adapted to meet the needs of immigrant populations, inform the design of an information resource about health insurance?

RQ3. What are the challenges and opportunities when engaging in community-based UX research projects?

In this paper, we situate the case with examples drawn from the literature related to communitybased research, specifically research about localization and usability studies with immigrant populations. We then discuss how this case was studied by describing the usability study used to investigate an information resource and describing our research site, a community health center serving immigrant and low resource clients. We present the case by articulating the problem: the challenge of enrolling multilingual audiences with diverse needs and abilities into healthcare under the Affordable Care Act. We provide an overview of the solution, a guidebook designed to help multiple audiences enroll and a usability study to assess how well the guide was working for 
two target audiences. We then share the process for developing the solution, the guidebook, and the study. We conclude by reporting on how a usability study, adapted for the needs of immigrant populations, impacted the design and iteration of the guidebook. The case study concludes with a list of its broader implications, specifically an examination of the challenges and opportunities for engaging in community-based UX projects. The case study provides insights to UX professionals and technical communicators looking to partner with organizations serving the community and wanting to adapt UX practices to be more appropriate and suitable within nonprofit organizations.

\section{SITUATING THE CASE}

In this section, we situate this case study by identifying themes in related scholarly and practitioner literature. First, we detail how the literature was selected. Second, we define the terms we use throughout the case, including user experience, usability, and community-based research. Finally, we identify other related studies and identify three themes from the literature that apply to this case.

How Literature Was Selected Because this interdisciplinary case crosses the domains of user experience, health information, and immigrant populations, we conducted a broad search of the literature to identify related cases. We searched comprehensive multi-disciplinary databases, such as Academic Search Complete, ACM Digital Library, and Google Scholar, and used key words such as "community-based research," "user experience," "usability," "immigrant populations," and "health insurance information design."

Defining User Experience and Usability User experience, according to ISO 9241-210, is “a person's perceptions and responses resulting from the use and/or anticipated use of a product, 
system or service" [3] and refers to both the process of designing software and systems and the outcome of the interactions with that system [4]. People working in UX come from a variety of backgrounds and disciplines, including anthropology, psychology, computer science and technical communication [5]. Technical communication has had an intertwined history with UX [6] and the skills that technical communicators possess overlap with those required in UX positions [7].

A key component in designing and evaluating user experiences is conducting applied research, including usability studies that evaluate how well a product meets the needs of users [8]. How a usability study is conducted depends are diverse and diverge based on the goals of the study and the needs of the organization. Usability studies can be formative or summative, remote vs. in person, moderated or unmoderated [8]-[10]. The choice of what usability method to use to evaluate a design is contingent upon a variety of factors, including budget, schedule, organizational support, context of use, and the kind of data needed to answer the research questions (attitudinal vs. behavioral, qualitative vs. quantitative) [10]. In addition, usability studies are part of a larger set of applied research methods to choose from during the design process and are contingent on factors such as context of use and the type of data needed to answer the research questions [11].

Community-based research consists of the relationship between researchers and community members or partners who are pursuing questions of mutual interest and sharing power and decision making [12], [13]. Technical communication has a long history of engaging in projects with communities and organizations. Examples of this type of work include service-learning 
projects [14]-[17], client-based student projects [18], and projects supporting and examining civic discourse [19], [20].

Related Studies Three studies informed this research project. The first, from Walton, Zraly, and Mugengana, presented insights into their process for navigating the messiness of a communitybased research project in Rwanda [13]. The second is Agboka's study of participatory localization that foregrounds a social justice approach [21]. The third study, from Bobeth et al., discusses methodological experiences with user-centered design to design with and for immigrants [22].

Walton et al. characterize community-based research as "unpredictable, mutable, contingent, serendipitous, complex, and challenging." [13, p. 45]. In their study, Walton and her co-authors provide insights from a community-based multilingual study conducted in Kigali, Rwanda, where they incorporated an explicit focus on values and validity to inform the uncertainty and challenges of community-based research. Their study highlights both the complexity of community-based research and the value of shifting the balance of power towards the community partner. They also conceptualize rigor as being specific to the site, strengthened through triangulation of multiple methods, and based on the input of community partners. Walton et al.'s study explores the fact that flexible methods can retain a high degree of rigor and the importance of power sharing with community-based partners.

Agboka's study of participatory localization is motivated by a commitment to social justice by examining ways to improve localization practices at international sites [21]. His study investigated the localization efforts of designers on marketing documentation of sexuopharmaceuticals imported from China to Ghana. Instructive in Agboka's article is the 
concept of "participatory localization," which expands a more traditional notion of user-centered design from an individual focus to a community focus. As he states, "This means that we need an ethnographic, participant-observer approach to the localization process that requires careful, humble, and thorough outsider involvement with local users and in which users and designers co-construct knowledge.” [21. p.43]. Simply translating documents does not take into account the cultural and legal issues situated in a specific locale. Further, Agboka's model of participatory localization requires the participation of stakeholders at the local level because "a one size fits all model" would not be appropriate or adequate. He goes on to outline five paths to guide localization. First, localization should take place locally, at the users' site. Second, it should be a collaborative effort between users and developers. Third, localization and translation are not the same. Fourth, localization needs to be based on prolonged engagement and be an ongoing process. Finally, localization should be based on its relevance to local needs.

The third study, from Bobeth et al., presents methodological reflections related to engaging in design with and for Turkish and Arabic immigrants in Europe through the user-centered design process to develop supportive information and communications technology services for smartphones [22]. In their study, they engaged in a variety of methods, including developing a list of barriers and conducting semi-structured interviews, focus groups, and participatory design workshops. Their discussion and the implications of their study proved instructive for our study. A main theme for their research was a close collaboration with NGOs, due to these organizations' deep knowledge of the immigrant communities and their established relationships, which engendered trust for the research. They also learned about the benefit of conducting research in the "mother tongue" or native language of the participants. 
Themes in the Literature From our review of the literature and the related studies, three key themes emerged to inform our study and the notion of community-based user experience. First, participation in community-based research needs a strong partnership with a community organization. Second, translation and translators have a critical role within the research project. Third, the data and insights gathered in a usability study need to be valuable for the organization to inform design.

The first theme from the related cases is the notion of participation in community-based research. Participation typically entails direct engagement between the researchers and community members throughout the research process to share power and decision making. Within the traditions of UX, various notions of participation and representation have been deployed in different approaches. In the practice of participatory design [23], [24], tracing back to its Scandinavian roots [25], participation is conceived as full engagement by members of the community throughout the entire design process. In contrast, user-centered design, emerging from a North American tradition [26], [27], espouses a more representational notion of participation; users engage with research (in surveys, usability studies, interviews) but not with full participatory status as members of the design team [28]. Within this project, we draw on the philosophical commitments of community-based research, while also adhering to the constraints of our community partners to create meaningful research results. Therefore, we conceptualized participation in two ways. The first was the full participation of a nonprofit community partner, the Community Health Center staff, who shared decision making about the research questions, methods, data, and results. The second was the representational participation of the patients who were the target users of the design. This choice was informed by the input and needs of the nonprofit organization. The need for a close partnership is mentioned by Walton et al.[13] and 
Bobeth et al.[22]. Based on our positions as outsiders to the community, we needed to engage closely with the nonprofit to legitimize the research, engender trust with participants, and ensure that the results were meaningful and relevant to our community partner. It was only through the collaboration that the research was possible, and it was more appropriate in this case to engage the immigrant populations through the mechanism and existing structures of the nonprofit.

The second theme from the related studies addressed the importance of translation and the role of translators. Walton et al. identify the importance of engaging translators in the community-based research project as active participants and note that translators are, according to Larkin et al [29] "co-constructors of meaning" [13, p. 49] and what Temple and Young [30] call "cultural brokers" [13, p. 49] who "always shape data in cross-language research" [13, p. 50]. Bobeth et al. also talked about the importance of the role of translation within their user-centered design project with immigrants and the importance of engaging translators who are from the same country of origin as the participants [22]. Finally, turning to the translation of an information product, Agboka reminds us that the issues of translation are not solely rhetorical, but also situational, and that regarding language, "One can argue that, even at the basic level, the same languages differ based on context and geography" [21, p. 45].

The third theme in the studies was the importance of shaping the research so results are relevant to the local community and context [13], [21]. Walton et al. state that the challenges of doing community-based research are not signs of a lack of rigor; instead, "well-designed, wellconducted community-based research encounters unexpected challenges and serendipitous surprises because power is not centralized with researchers and because complex, dynamic local contexts are informing the work" (p. 62). This theme informed our case in the ways that we 
negotiated the research project, designed the study, and shared the research results. While traditional community-based participatory work engages community partners throughout, we were also mindful of our community partner's constraints, namely time and resources. Our goal was to partner on a rigorous and informative usability study that provided valuable insights for our community partner.

\section{How This Case Was STUdied}

The purpose of this section is to provide insight into how the case was studied. First, we provide an overview of the research partner and participants and detail the research design. Next, we discuss how data were collected and analyzed. Finally, we consider credibility and trustworthiness.

Research Partner and Study Design Our partner in this case study is a community health center called International Community Health Services (ICHS), a nonprofit organization providing health services in and around Seattle, Washington to the broader community with a specific focus on serving Asian, Native Hawaiian, and Pacific Islander communities. In 2014, the year we engaged in this project, ICHS served more than 21,000 patients and enrolled 10,000 patients into the Affordable Care Act.

Our engagement with ICHS came about due to an existing connection between one of the authors of this article and the staff at the nonprofit organization. The first two authors of the article are experienced UX professionals and university academics with expertise working with nonprofit and social impact organizations. As researchers, we were curious about how the organization was changing and adapting to the implementation of the Affordable Care Act (ACA). After informal conversations with ICHS about the topic of enrollment in the ACA, we 
engaged more formally through a series of phone calls and meetings. As UX professionals experienced with community-based research projects, we approached these initial conversations to understand the challenges that the organization was facing and to explore how our UX experience could support or supplement the organization's efforts during ACA enrollment. In other words, we approached the engagement by asking "How can we help?"

ICHS possesses some of the hallmarks of nonprofit organization: it plays a unique role in the community, shifting and moving to fill gaps in infrastructure and services, and it faces numerous constraints, including limited resources, need for compliance with regulations and policies, and a fluid and dynamic workforce [31]. Therefore, during the engagement, our ethical stance was to act in a helping and supportive role in the collaboration, while being sensitive to the organization's constraints. As we engaged in the project, a primary constraint was ICHS's limited time. ICHS is a busy clinic serving a growing constituency and we were embarking on this project during insurance open-enrollment, one of the clinic's busiest times.

Because this was a community-based research project, we negotiated the focus and methods with our partners at ICHS by reviewing existing documentation about the first year of the ACA enrollment process year [32]. In addition, we had several scoping conversations via email and in person meetings with the staff. To maximize the benefits of our collaboration while respecting the time constraints of our community partner, we mutually agreed on a scope and study design that the organization perceived would offer the largest benefit for their existing needs. As a result, we collectively decided to conduct a usability study on an existing supplemental guidebook that the clinic developed to support patients in the enrollment process. More details 
about the rationale for developing and evaluating the guidebook are detailed below in the About the Case section.

Conducting usability studies was new to ICHS. As we defined the research, we were also in the position of explaining and justifying how we would conduct the research. A usability study is an applied research method that aims to understand the use of information or technology to make improvements. In a usability study, representative users attempt to complete realistic tasks while an interdisciplinary team collects empirical data and analyzes that data to make improvements [8]. The representative users for this study are described in the Participants section below. The focus of the study was articulated in five specific areas:

- Reader's goals: Does the guidebook help readers answer their questions related to insurance plans?

- Comprehension and translation: Is the content comprehensible and understandable for the audience?

- Organization: How well does the organization of the guidebook help people determine their insurance options?

- Design and layout: Does the signposting in the document help users understand and navigate the information?

- Specific content areas: What specific areas, concepts, content, and terminology do users struggle with?

These areas led to the creation of a series of tasks that participants would attempt during the usability study (see Table I). These tasks were developed collaboratively and included common questions patients have when signing up for health insurance, as well as concerns and questions 
that the team had about the design of the guidebook. The research protocol for the study was reviewed by the University of Washington's Institutional Review Board and also went through an internal research review by our partner organization, ICHS.

Participants To evaluate the guidebook with representative users, our team developed recruiting criteria to identify potential participants. The following criteria for inclusion were used:

- Existing or prospective patients seeking health care at International Community Health Services (ICHS) clinics

- Speakers of either Cantonese or Vietnamese, which are the languages spoken by the two largest language groups served by the community health clinic [32]

- Patients who were currently uninsured or planning to enroll in insurance in the upcoming enrollment period

Participants were recruited by in-person assistors (IPAs), who are staff at ICHS and regularly meet with patients to help them sign up for health insurance. IPAs told prospective participants about the study at the end of a health insurance appointment. Potential participants called a phone number to express their interest and provided their contact information. A study facilitator called, screened, and (for those who were eligible) scheduled potential participants. Some callers also asked whether they could refer friends to take part in the study, and we encouraged this type of snowball sampling [33]. Each participant was given a US $\$ 50$ gift card to a grocery store for their participation.

According to best practices in the usability literature, five users will find $80-85 \%$ of usability problems [34]. We recruited a total of 12 participants: six who spoke Cantonese and six who 
spoke Vietnamese (see Table II). This number is in line with best practices in usability testing in formative studies that focus on finding problems [10]. All participants in the study were current or prospective patients at ICHS, native speakers of their language subgroup, and immigrants residing in the United States. All participants had a range of language abilities, and some had limited understanding and fluency in English. All study sessions were conducted in the participants' native languages. While we attempted to recruit an equal distribution of genders across the study, we were not able to achieve this goal.

Data Collection To conduct the usability study, we recruited two facilitators to the research team from our university. Each was natively fluent in one of the target languages, was also fluent in English, and had experience with usability studies and research. The facilitators translated the study materials, including consent forms, recruitment information, and scenarios, and also conducted the studies in the participants' native languages. Aware of the methodological challenges related to conducting usability studies with populations who speak different languages, we made appropriate modifications to the facilitation style, making it more conversational than a traditional study [35].

The study was conducted over the span of two days at the ICHS clinic in Seattle, WA in December 2014. Each session lasted approximately one hour. Participants were given an overview of the study's purpose and the use of the data, and an explanation of informed consent. Participants were given between five and seven tasks that asked them to look for information, interpret insurance details, and provide feedback on the guidebook. The tasks were developed collaboratively with the nonprofit staff and are shown in Table I. 
To collect data, facilitators used a think-aloud protocol [36], a best practice in usability studies [8], [10]. However, we discussed as a research team the limitations of this approach. First, the document being tested was a guidebook used in conversation between clinic staff and patients. Therefore, asking participants to speak as if the facilitator were not there created an inauthentic experience for participants. In addition, the team thought that the traditional think-aloud protocol felt culturally inappropriate and somewhat rude. They were concerned that the participants might feel as if they were being quizzed. Therefore, we modified the traditional protocol to be more conversational in nature. Because the intent of the study was to discover the document's comprehension challenges, we had hoped that a more conversational style would identify the document's problem areas and the content that was confusing. Our goal was not to assess or evaluate the nuances related to the comprehension problems during the study itself but rather bring this descriptive data back to the community partner for further discussion.

As the study was formative in nature and designed to inform the next iteration of the document, we focused on issues of confusion and comprehension over task completion or success. Therefore, we chose not to collect more quantitative usability study metrics such as time on task, success rate, and so on. Instead, we identified issues related to comprehension or confusion, and logged each issue individually. These modifications to traditional usability protocols are examples of localizing the usability methods to make them appropriate to the context [35].

Each facilitator conducted the study in the participant's language and also took notes in that language. In other words, they did not translate the notes into English but rather kept all conversations and notes in either Cantonese or Vietnamese. Each session was video-recorded, but only the audio, the document, and the participants' hands were captured to preserve their 
privacy. The other researchers were also present in the room during the study to assist with the technical aspects of the study and take notes. We wanted to take notes on body language and record how the participants were navigating the documents. However, without ability in the participant's language, these notes were limited.

Immediately after each session, the facilitator and the other two researchers would debrief on the study results in English. Each debrief lasted 30-45 minutes, and the team stepped through the entire study with the facilitator, reading from their notes and sharing key quotes and areas of struggle from the session. As a group, we discussed the major issues for each task and captured them thematically in a structured document. While it would have been optimal for the nonprofit staff to observe the usability sessions, this was not possible due to logistical constraints, such as space and staff availability, and linguistic constraints, since the sessions were conducted in the language of the participant. However, twice during each study day, the research team checked in with ICHS staff and shared emerging findings, clarified questions, and discussed areas where participants experienced comprehension problems.

Data Analysis After the conclusion of the study, the research team analyzed their notes of the debriefing sessions. First, the team analyzed each participant's experience to identify comprehension issues. Second, the team went page by page through the guidebook to visually identify any areas of comprehension problems as a second pass. Finally, the team returned to the main areas for the research study and looked thematically across all of the issues identified across the participants to identify larger themes. Because the study aimed to understand comprehension, any issue that arose was added to one of the themes if it was salient to the design. 
After the study, the two primary authors reviewed all of the notes taken during the study and refined the list of findings. When areas needed additional clarity, they conferred with the two translators to examine the issue in more detail. The results of the study were then captured in a presentation slide deck to share with the broader team.

Ensuring Credibility and Trustworthiness To discuss the credibility of the research, we will detail the measures we took in terms of both usability studies and also community-based research. For usability studies, we used Hughes's guidelines for qualitative research as a guide to ensure rigor [37]. These included ensuring that the scenarios reflected the users' perspectives and were vetted by proxy members of the user community (in our case, the nonprofit staff). We used a modified think-aloud protocol to record users' direct impressions. We also selected participants based on criteria that reflected the product's end users and used real versions of the product in the study.

Further, during the usability study we aimed to ensure credibility and trustworthiness by conducting frequent debriefing sessions [38] between the UX team and the nonprofit staff. These debriefing sessions occurred informally between study sessions, at the end of each day of the study, and then at a briefing meeting to share the emerging findings from the study. These sessions helped both to inform everyone of the emerging findings and also to gain insights and reactions from the internal team and their interpretations and elaboration of the data as they were being collected.

In terms of validity for the community-based research approach, we worked with our community partner to foster a relationship built on mutual respect and co-ownership of the research project. Finally, as our team presented the research results, we did so in a way that invited dialog and 
collaboration and allowed us to share and make meaning together. To do so, we started with a broader discussion of how the document was working during enrollment and what ICHS staff had learned from using the document with patients. Presenting the research in this way helped to open the dialogue and privileged the staff's local knowledge. In a way, this approach helped to shift the usability results from one input of insights and instead triangulated these findings with what the local knowledge and experience of the nonprofit staff working with patients. In addition, we chose to present the usability study results as findings and open-ended recommendations, instead of as firm recommendations of what to change. Then we brainstormed as a group the possible ways to improve the usability of the guidebook. This process allowed us to negotiate as a group based on overlapping areas of expertise in document design, usability, health insurance, and knowledge of patient needs.

\section{About The Case}

In this section, we provide an account of the engagement between UX practitioners and a nonprofit organization to understand the challenges confronting patients signing up for health insurance and to evaluate a guidebook through a usability study. First, we present the problem and then the solution.

The Problem For many people living in the United States, obtaining health care and health insurance can be a challenge. This is especially true for immigrant populations. Many immigrants in the United States turn to community health centers for care. Community health centers are clinics that provide healthcare to medically underserved populations regardless of their ability to pay for services. 
In 2013, the US government implemented the Affordable Care Act (ACA), a comprehensive set of healthcare reforms that aimed to improve healthcare quality, lower costs, provide new consumer protections, and increase access to health care for all. The ACA increased funding to support and expand community health centers; as a result, they became central in the efforts to help people sign up for care and insurance [39]. Some of the patients who visit a community health center may not have access to technology like computers to sign up, and may not be familiar with insurance rules and regulations. In addition, patients may not speak English and may have different levels of reading literacy. To mitigate the challenges of signing up for health care, federal and state governments established in-person assister (IPA) programs to help people, particularly members of hard-to-reach populations, to learn about insurance, enroll in plans, and address issues that arise after they've been enrolled in their insurance [40].

ICHS, a community health center in Seattle, Washington aimed to support patients signing up for health insurance by designing a guidebook specifically for their patients. After the initial enrollment period in 2013-2014, ICHS conducted an assessment to examine the challenges associated with signing up patients for insurance and concluded that patients needed supplemental information in their own language that could act to bridge the information gaps, improve understanding about the ACA, and as a result, improve the enrollment process for staff and patients alike.

The Solution The guidebook's purpose was to provide patients with supplemental information to inform them about health insurance options. Further, its specific design goals were to improve the enrollment process, answer patients' common questions, and act as a reference outside of appointments to take home or share with others. The audience for the guidebook was ICHS 
patients enrolling in a health insurance plan for the first time. Using data tracked during the initial enrollment period, ICHS identified their patients' top three preferred languages as Cantonese (41\%), Vietnamese (21\%), and English (18\%). Therefore, ICHS created one guidebook in English and translated it into Cantonese and Vietnamese for the 2014-2015 enrollment period.

Brief Description of the Project The guidebook was designed by ICHS staff and the information and design choices in the guidebook were inspired by the IPAs' experiences with patients. After the guidebook was designed, a group of external technical communicators designed and conducted a usability study with the target audiences of the guidebook. The study yielded rich data about the participants, their information needs, and the design of the guidebook itself. After the study, the UX team and ICHS staff met to debrief on the study results, discuss the findings and brainstorm solutions. Based on the study results and additional feedback from staff during the second enrollment period, the ICHS staff redesigned the guidebook.

Process for Developing the Solution In this section, we provide information about the process for developing the solution organized into three milestones: developing Version 1, conducting a usability study, and suggesting changes to Version 2.

Version 1 of the guidebook was developed in response to challenges related to the inaugural enrollment period of the Affordable Care Act enrollment in 2013-2014. ICHS conducted an internal evaluation of the first enrollment period and identified a variety of challenges and potential solutions to improve enrollment, including health insurance literacies, the lack of culturally and linguistically appropriate resources, and technological barriers. This information 
answered our first research question: What are the information needs and barriers faced by immigrant populations signing up for health insurance?

Health insurance literacy became crucial with the implementation of the ACA because consumers, many of whom were currently uninsured, needed to understand and choose a health insurance plan. In 2012, the Consumer Union identified three broad skills that encompass health literacy: understanding what the features of health insurance are, how to purchase or select health insurance, and how to use it [41]. Also, many people struggle to use income tables to determine their insurance eligibility [42], lack the self-confidence to choose health insurance on their own [43], and possess low numeracy skills that hinder their ability to understand or choose between different health insurance plans [44].

For ICHS, the issues of health insurance literacies were not confined to the patients. The complexity of health insurance also challenged the IPAs designated to assist them. The implementation timeline of the ACA meant that patients had only a short time to learn about insurance and new insurance concepts, determine their eligibility, select a plan, and enroll. This process was complicated because patients needed to understand their complex tax and immigration statuses as non US-citizens to know what kinds of plans they were eligible for. Even some IPAs thought that they did not have enough knowledge themselves to help patients navigate the complexities of plans. As a result, ICHS concluded that patients needed supplemental information, in their own language, that could bridge the information gaps and improve understanding about the ACA, and as a result, improve the enrollment process for staff and patients alike. 
ICHS ultimately created guidebooks in English, Cantonese, and Vietnamese. The information was first developed in English by the staff and went through several iterations to refine the information, context, design, and messaging. The text, along with the document's visual layout, was designed to be concise, simple, and appealing for patients. Once the document was prepared in English, it was then translated by IPAs who had both domain knowledge and fluency in linguistic and cultural practices of the target audience.

\section{RESUlts}

In this section, we describe findings related to our second and third research questions. We first present the findings from the usability study as four themes and report their implications for the design of the guidebook. Then, we discuss the opportunities of engaging in community-based UX research projects.

Findings As with most usability studies, we learned a great deal about how the product was serving users and their needs. Here we provide the four themes that are most germane to the case study and salient to the design. We also provide visual examples and, when possible, emblematic quotes from study participants. The four themes from the study are the following:

- The positive value of the guidebook and issues of trust

- Challenges in understanding eligibility and plans

- Challenges with health insurance terminology and costs

- Issues of uncertainty and anxiety

The Positive Value of the Guidebook and Issues of Trust Overall, the guidebook was positively received by the participants. During and after the study, several participants commented 
positively about the guidebook's value. They found it to be a valuable resource, and some had brought their own copies to the study. One participant said, speaking of his experience, "Because our English is not good ... we depend on people for help. But because [the guidebook] is good, I would take the initiative to look at the information." The participants in our study also had an existing relationship with ICHS and looked to the organization for help. This relationship translated into trust in both the organization and the guidebook. Participants anticipated and felt confident that the guidebook will help answer the questions they have. However, some participants still wanted to have conversations with staff. They expressed suspicion about translation based on previous experiences with documents being poorly translated. These participants who expressed concerns about translation appreciated the materials but still wanted to have a conversation in person with someone who spoke their language.

Challenges in Understanding Eligibility and Plans Whether community members are patients or not, they can visit the ICHS clinic to get help signing up for a health insurance plan. However, a variety of complex factors impact which health insurance plan they may sign up for, including length of time in the US, income, and size of household. The guidebook was designed to help patients navigate the plans for which they qualified by providing income charts, flowcharts, and details about the levels of plans.

Overall, we found that all of the participants in the study struggled to understand the requirements for qualifying for plans and the methods for paying for these plans. In one of the study tasks, participants were asked to determine health insurance for a friend. The guidebook provided two resources to help determine eligibility, the first a chart showing income based on Federal Poverty Level (see Figure 1), and the second a set of flowcharts showing different 
eligibility scenarios (see Figure 2). In the study, participants struggled to use these two information resources and interpret the information necessary to successfully complete the task.

There were a variety of reasons that participants struggled with the task. The Federal Poverty Level chart is pictured in Figure 1. The columns in the chart indicate different percentages of poverty level income and the rows indicate household size. Several participants remembered this chart from their interactions with IPAs, but had difficulty comprehending it unassisted. While some participants were able to find their income levels, some did not understand the significance of the different columns. In addition, the word "household" on the chart was embedded in the graphic and not translated into the target language, which further confused some participants. One participant said, "This is the poverty line," pointing to the row lines in the document. She continued, "If you are listed you are poor," mistakenly concluding that all of the amounts on this chart mean you qualify for services.

The flowcharts were also challenging for participants to use (see Figure 2). One participant articulated that the design of the flowcharts was "overwhelming with all the arrows, all you see is blue and red. I had to calm down to follow the text." The guidebook uses images that show abstract images of people with minimal details, but enough to convey either age or gender. This confused some participants who thought the difference between the flow charts was gender rather than age. The flowcharts also use terms and information from other places in the guidebook, such as the Federal Poverty Level percentages, but they do not directly reference the location of that information, so the participants weren't sure where to get information about the income levels or what the numbers in the flow charts represented. 
Participants struggled to reconcile their specific questions and needs with the examples provided in the guidebook. For example, patients can choose among three Qualified Health Plans (Bronze, Silver, Gold). To show the difference in cost between the plans, the guidebook used the Silver plan as an example (see Figure 3). Participants again struggled to understand key insurance terminology and did not clearly understand how the three plans differed. Several participants mentioned they knew that the costs would be different, but how they would differ and what the right choice would be was not apparent. One participant concluded that the Silver plan shown as the example was the "standard" or "recommended" plan. Others said they didn't know the differences among the plans, and one participant said she didn't care to know. Because the examples in the guidebook did not address a participant's specific situation, participants did not find the information relevant.

Challenges with Health Insurance Terminology and Costs Participants struggled with a variety of key concepts and terms related to health insurance information, particularly that related to purchasing and payments. The relationships among deductibles, premiums, and out-of-pocket maximums were also confusing to many participants. Trying to understand the range of costs associated with health care insurance can be a challenge.

The findings in this study echo Blumberg et al.'s survey that demonstrated comprehension related to insurance terms is poor among many Americans is low and this is especially true for those who are new to health insurance and lower-income adults [45]. Participants were given a task related to shopping for insurance plans and asked to define, in their own words, key terms like deductible, premium, co-pay, co-insurance, and out-of-pocket maximum. While some 
participants successfully defined some of the terms, very few were able to define all of them, suggesting that they had a partial or incorrect understanding about the cost of their insurance.

To help readers understand health insurance terms, the guidebook provided a glossary on its last page, which was rarely used during the study. When the glossary was used, participants still struggled with some of the terms. The term "deductible" was not easily understood by most participants. Several participants saw the deductible as "a deposit," something that you had to pay up front, before you received care. Others stated that it was a monthly payment, perhaps confusing it with the term "premium." Still, others stated that the deductible was money you have to pay over the course of a year for your health insurance.

Issues of Uncertainty and Anxiety The two previous themes related to specific comprehension issues related to the complexity of health insurance, including eligibility, plans, terminology, and costs. Participants brought the implications of these challenges to life by highlighting how these issues caused them to feel uncertainty and anxiety. Because of the conversational style of facilitation, participants shared their struggles with the concepts of health insurance and the ways that they impacted their lives, including major life decisions and financial planning. Several participants talked about how choices related to health insurance had an impact on major life decisions. For example, one participant mentioned that his family was trying to decide whether an elderly parent might move out of the family home, and if so, whether that change would impact the rest of the family's eligibility for a subsidized health insurance plan. Another participant mentioned that she felt that she couldn't change jobs because of the impact on her eligibility for health insurance. 
Participants wanted to have a clear sense of how the cost of health insurance and health care would impact their budget so that they could prepare and save. However, the level of detail needed to convey this information was not possible to provide in the guidebook and, in a sense, never really possible due to the unanticipated or unexpected costs associated with health care within the US healthcare system. These stories, in light of the comprehension challenges, illuminated how challenging it was to develop an information resource that could inform patients about health insurance, mitigate their anxiety, and prevent misinterpretation.

Design Changes Based on User Feedback In this section, we discuss how the four findings from the study led to changes in the design and the use of the guidebook. We have organized these implications into two themes, first, the role of the guidebook in supporting the in-person experience, and second, specific design improvements. As mentioned in the methods section, the UX team first analyzed the data from the study and then met with ICHS staff to discuss the study findings and collaboratively brainstorm solutions. The UX team documented the study results in a slide deck. Rather than a formal presentation, the results were shared in an interactive dialog. In addition, the study had taken place during an active enrollment period, so while the UX team had been conducting the study, the ICHS staff had been using the guidebook with patients. Therefore, the study results often confirmed or elaborated on the issues that the ICHS staff had been experiencing with patients.

The Role of the Guidebook in Supporting the In-Person Experience While the ICHS staff had always conceived of the guidebook as a supplement to in-person meetings with patients, there was also a hope that the document might be used outside of appointment times either by the individual patient or by family or friends in their social network. The results of the study pointed 
out a variety of places within the guidebook where patients were at best confused and at worst coming to incorrect conclusions based on their interpretations. In addition, we discussed the anxiety and issues associated when patients had misinterpreted information about health insurance and how, in order to continue to engender trust, the relationship between clinic staff and the patient needed to be the primary focus.

Therefore, instead of attempting to collect the expertise of clinic staff and represent it within the guide, we discussed how the guide could support the in-person experience rather than replace it. This acknowledgment allowed the team to brainstorm how the guidebook could be improved and function within the larger ecosystem of services that patients encounter in the clinic. As a result, in Version 2, the guidebook was streamlined to remove some of the more complex content and instead shift the more complex tasks to the in-person visit. Some of these specific recommendations are detailed below.

Specific Design Improvements To improve the design of the guidebook, the team made several key decisions aimed at supporting patients' key questions and reducing visual complexity. First, instead of refining confusing information, the team chose to remove information that was better discussed during in-person meetings. For example, this meant removing the flowcharts altogether from Version 2.

In addition to removing content that was problematic for patients, Version 2 was redesigned to reduce visual complexity. Version 1 of the guide used a variety of colors and abstract images to enhance its visual appeal. Several of the usability issues in the study were either traced to or exacerbated by this visual complexity. Therefore, in Version 2, visual enhancements that were not directly linked to the content were omitted to simplify the guidebook's overall design. For 
example, the original income table picture in Figure 1 was redesigned to simplify the information, a design change that removed the color and the 3-D effects (see Figure 4).

Finally, an additional significant change to Version 2 was to reorder the information in the guidebook to map to patients' key questions and patterns of looking for information. As a result, the glossary, which users had found useful in the study, was moved from the last page of the guidebook to the second page. While conventionally, readers might expect a glossary at the end of the document to serve as a reference, in the study we found that it had mostly been overlooked. However, when users did find the glossary, it provided a valuable orienting tool with definitions for the various insurance terms used throughout the guidebook.

UX Projects in Community-Based Research Because community-based research projects are highly localized and therefore applicable to a particular site, we conclude with some broader reflections on the project to share with others doing community-based UX projects to address the final research question in this case study: What are the challenges and opportunities when engaging in community-based UX research projects? Here, we present three implications that we hope that others working on similar projects will find useful, and summarize the characteristics of teams engaged in community-based UX projects.

First, community-based UX collaborations have the ability to leverage and therefore amplify multiple types of expertise. Community-based organizations have deep expertise within their domains and extensive knowledge of their target users. Unlike large organizations that may interact with customers only at a distance or through service channels, many nonprofits such as ICHS have existing and intimate relationships with their users - in this case, their patients. The staff was knowledgeable and experienced about their patients' culture, needs, struggles, and 
challenges related to insurance because the relationship between staff and the community was already quite close. Therefore, when the UX team had questions about user expectations or existing knowledge, the ICHS staff were prepared to answer these questions comprehensively.

Complementing the organization's knowledge of their audiences was the expertise that the UX team brought to the collaboration. The UX team's skills in conducting practical and actionable research in the form of a usability study were new to this organization. In addition, the study was conducted quickly and inexpensively. The only cost was the honoraria for participants that the researchers provided based on funding from their academic institutions. In addition, the UX team used the results of the study to identify what about the design was working, what might need improvement, and how the guidebook could be positioned within the information-sharing relationship between IPAs and patients. What worked well was the two teams coming together with their unique perspectives and expertise to focus on the problem and develop possible solutions.

Second, UX practices need to be adapted to be appropriate for the particular setting and audience. Based on our previous experience conducting research in international settings (see [35],[46]) the UX team was prepared to modify the methods as necessary for the setting. The usability study using translation, and the translators also played a lead role in interpreting the issues that users faced during the study. While we discussed using standard usability metrics, such as time on task, task success ratings, and the System Usability Scale, we decided on a more formative, open-ended style for the study that focused on barriers and areas of confusion.

We conducted the study this way for a variety of reasons. First, because the study was facilitated in the native languages of the participants by the translators, assessing task success and 
completion was difficult to do in real time. Because the study results had to be turned around quickly and because the budget was limited, we were not planning to have the study sessions transcribed and translated. Therefore, instead of focusing on task completion, we focused on issues of comprehension instead. Second, the topic we were investigating was the content related to signing up for health insurance, a complex task for many people, especially with a new system of insurance and participants who were not previously familiar with the concept. Therefore, the completing the tasks was not always straightforward, and hearing the questions and struggles that participants experienced while trying to complete the tasks shed insight into common areas where patients struggled to understand concepts. Finally, we decided to elicit the data in a more conversational style, rather than a traditional think-aloud protocol. We made this decision to reduce the burden on the facilitator, to better engage with the participant during the study, and to collect data that were nuanced and could facilitate an open discussion with ICHS staff, rather than to deliver a firm and hard set of metrics to report. We acknowledge that there is a limitation to capturing data in this way. For example, we sacrificed collecting comparable baseline metrics that could show the impact of the changes to the design over time. Gaining timely actionable outcomes took precedence over developing a more sustainable and quantified UX practice.

The usability study did not provide the full experience of how users were able to use the guidebook, but it did pinpoint and predict some specific areas to improve. The data from this study, combined with stories from internal staff using the document, triggered the changes in Version 2 of the design. It was important to set these expectations for both sides - for the UX practitioners to realize that changes would be incorporated whenever possible and for the organization's staff to realize that the feedback and recommendations were a partial understanding of how the document actually worked. This practical approach allowed us to do 
what was possible with few resources. This combination of community and UX expertise can provide a powerful set of skills to create audience appropriate information.

\section{CONCLUSIONS, LimitaTIONS AND SUgGESTIONS FOR FURTHER RESEARCH}

This project, a collaboration between a community-based nonprofit organization and a team of UX professionals, provided a rich site of learning that resulted in an iteratively designed guidebook that was evaluated with target patients. In this section, we conclude with some thoughts about community-based UX projects, reflect on limitations, and offer several suggestions for further research.

Conclusions Practicing UX is inherently highly nuanced and rhetorical [47]. However, the main takeaway from this work for UX professionals is that working in a community-based context requires humility. To share power with community partners, the choice of UX methods and the engagement itself need to be flexible and customized based on the partner's needs and constraints. As a result, it may be necessary to modify methods or, as Buley recommends [48], adhere to "principles over process" where inviting others in, truly listening, and working towards "good enough" can be more beneficial over trying to adhere to a more corporate or standardized view of UX and UX practices.

Community-based UX collaborations can amplify multiple types of expertise. However, UX methods may need to be adapted to be appropriate for community-based collaborations that incorporate local knowledge and needs. UX and nonprofit staff have complementary and overlapping areas of expertise, as shown in Figure 5. UX practitioners have developed skill sets to understand users, including a variety of tools and methods in applied research aimed at audience analysis. UX practitioners also have expertise in understanding how systems work, how 
information flows through those systems, and where the human touch points that occur along the way are located. UX practitioners are also steeped in evaluation methods such as usability studies and heuristic evaluations, which enable a data-driven perspective for making decisions about design. Finally, iterative design is central to UX practice and acknowledges that a feedback loop and multiple drafts are required to create a successful design.

Nonprofit staff have unique and complementary expertise that strengthens the collaboration. They have deep knowledge of both the domain —in this case, health care and health insuranceand the constituents or patients that they are serving. Nonprofit staff also understand the cultural context and the community that they serve. In our collaboration, the nonprofit was embedded within the community and possessed a level of trust and access to community members because they are a part of the community they serve. Finally, the nonprofit staff we worked with had a clear understanding of information design and also of the appropriate channels to disseminate information to their constituents.

While each set of professionals has unique expertise, both share common values. Both pursue a human-centered approach in their work. We intentionally use "human-centered" rather than the more common "user-centered" used in commercial settings, to adhere to Buchanan's definition that amplifies a focus on human dignity [49]. Human-centered design is fundamentally an affirmation of human dignity. It is an ongoing search for what can be done to support and strengthen the dignity of human beings as they act out their lives in varied social, economic, political, and cultural circumstances (p. 37).

UX practitioners and nonprofit staff also share an understanding of working in resourceconstrained contexts. Even within corporate settings, there is a pressure in UX to do more with 
less, as evidenced by the embrace of guerilla and lean methods [51]-[53]. Non-profit organizations also share the challenge of operating under funding constraints. This shared perspective results in a focus on both sides to stretch limited means - in other words we do what we can with the resources that we have.

Finally, UX practitioners and nonprofit staff both focus on problem solving. While nonprofit staff frequently focus on addressing structural inequalities or larger societal issues, they also need to be pragmatic, addressing real and current problems. UX practitioners who come from technical communication and engineering backgrounds tend to have an interventionist perspective, focused on solving existing and real-world problems.

However, in addition to the overlapping areas of expertise that act as a strength for these sorts of partnerships, community-based user experience projects also present various challenges. The first challenge, as previously mentioned and evident in our case study, is that nonprofit organizations have limited time and resources. They often do not have their own teams to focus on UX, and so any such activities are either add-ons to existing processes or simply not a focus. Introducing UX processes into an organization can be challenging and can require a variety of skill sets. In addition, when UX is new, organizational support can ebb and flow, depending on existing constraints and competing priorities [48]. Much of the work on nonprofits and UX focuses mostly on understanding users' experiences of websites and on encouraging charitable donations (see [2], [54]), rather than on the complexities of aligning UX practices with existing internal processes.

The second challenge is that findings from the community-based research project described in this study proved to be beneficial to the nonprofit, but it did not necessarily build what Dush 
refers to as rhetorical capacity that can translate to future projects [16]. In future engagements, we hope to create a more sustainable practice that could provide support and UX services to help build capacity.

Limitations The study was a community-based research project and collaboration between a UX team of professionals and the staff of a nonprofit organization. While we collaborated closely throughout the project, the project could have adhered more strongly to the participatory values of community-based research by engaging the nonprofit staff, or even the patients, in the actual design or facilitation of the usability study. However, the UX team found it difficult to ask nonprofit staff to take time away from their already busy schedules. The nonprofit staff was in the midst of an enrollment period and already quite busy helping patients sign up for healthcare, so they would have found it difficult to take time to be more involved in the study.

There were several limitations of the usability study due to the constraints on time and resources for the study. To conduct the study in two different languages, two different moderators facilitated the study. While study findings crossed both language groups, this difference in facilitation and translation may have affected the study results. The findings focused on the results across both groups rather than looking at specific issues for each cultural group. In addition, the lack of metrics reduces our ability to compare the two versions of the guidebook. Ideally, the redesigned guidebook should undergo a usability study to evaluate the changes with patients, but due to the lack of time, budget, and staffing, we were not able to engage in a second round of usability testing.

Suggestions for Future Research User experience practices can benefit all kinds of organizations creating information and communication technologies for their users. While many 
nonprofits are using these techniques, for others the practices are new or underdeveloped. In addition, there few studies reported in the literature specifically addresses the needs of nonprofit and community-based organizations as they deploy UX practices.

Specific research questions that could build on this case study include the following:

- How are UX practices in community-based organizations different from those in other types of organizations?

- What are some unique challenges and strengths of community-based organizations as they deploy UX practices?

- Finally, looking with a focus on community-based research and social justice, how can we as scholars, educators and students engage in cooperative, mutually beneficial projects? How can we sustain and cultivate these relationships over time?

For other UX and technical communication professionals interested in community-based research, we believe that our opening statement can be a good guide for this kind of engagement. Asking "How can we help?" opens the possibility for collaboration and cooperation that can benefit both UX practitioners and the nonprofit organizations that we aim to help. Within this dialog, we should also, as Agboka advises, approach this process with "careful, humble, and thorough outsider involvement with local users and in which users and designers coconstruct knowledge" [21, p. 43].

\section{REFERENCES}

[1] R. R. Johnson, User-Centered Technology. Albany: SUNY Press, 1998.

[2] L. Kruger, (2012) "Designing Nonprofit Experiences: Building a UX Toolkit," User 
Experience Magazine [Online] Available: http://uxpamagazine.org/designing-nonprofitexperiences/.

[3] N. Bevan, "International standards for usability should be more widely used," Journal of Usability Studies vol. 4, no.3, pp 106-113, May 2009.

[4] R. Hartson and P. S. Pyla, The UX Book: Process and Guidelines for Ensuring a Quality User Experience. Burlington, MA: Elsevier, 2012.

[5] J. Redish, "Technical Communication and Usability: Intertwined Strands and Mutual Influences," IEEE Trans. Profess. Commun., vol. 53, no. 3, pp. 191-201, 2010.

[6] J. G. Redish and C. Barnum, "Overlap, Influence, Intertwining: The Interplay of UX and Technical Communication,” Journal of Usability Studies, vol. 6, no. 3, May 2011.

[7] C. Lauer and E. Brumberger, "Technical Communication as User Experience in a Broadening Industry Landscape,” Tech. Commun., pp. 1-17, Jun. 2016.

[8] J. S. Dumas and J. Redish, A Practical Guide to Usability Testing. Exeter, UK: Intellect Books, 1999.

[9] C. Courage and K. Baxter, Understanding Your Users. Burlington, Mass: Elsevier, 2005.

[10] C. Barnum, Usability Testing Essentials. Burlington, Mass: Morgan Kauffman, 2011.

[11] C. Rohrer, (2014). "When to use which user experience research methods," [Online]. Available: https://www.nngroup.com/articles/which-ux-research-methods/.

[12] B. D. Faber, Community Action and Organizational Change. Carbondale: Southern 
Illinois University Press, 2002.

[13] R. Walton, M. Zraly, and J. P. Mugengana, "Values and Validity: Navigating Messiness in a Community-Based Research Project in Rwanda," Tech. Commun. Q., vol. 24, no. 1, pp. 45-69, Dec. 2015.

[14] S. A. Youngblood and J. Mackiewicz, "Lessons in Service Learning: Developing the Service Learning Opportunities in Technical Communication (SLOT-C) Database," Tech. Commun. Q, vol. 22, no. 3, pp. 260-283, Jul. 2013.

[15] J. B. Scott, “The Practice of Usability: Teaching User Engagement Through ServiceLearning," Tech. Commun. Q, vol. 17, no. 4, pp. 381-412, Sep. 2008.

[16] L. Dush, "Building the Capacity of Organizations for Rhetorical Action with New Media: An Approach to Service Learning," Computers and Composition, vol. 34, pp. 11-22, Dec. 2014.

[17] A. Mara, "Pedagogical Approaches: Using Charettes to Perform Civic Engagement in Technical Communication Classrooms and Workplaces," Tech. Commun. $Q$ vol. 15, no. 2, pp. 215-236, Apr. 2006.

[18] G. Getto, N. Franklin, and S. Ruszkiewicz, "Networked rhetoric: iFixit and the social impact of knowledge work," Tech. Commun, vol. 61, no. 3, pp. 185-201, Aug. 2014.

[19] K. Moore, "Exposing Hidden Relations: Storytelling, Pedagogy, and the Study of Policy,” J. Tec.Writing \& Commun., vol. 43, no. 1, pp. 63-78, Jan. 2013.

[20] W. M. Simmons and J. T. Grabill, "Toward a civic rhetoric for technologically and 
scientifically complex places: Invention, performance, and participation," College Сотр. \& Commun., vol. 58, no.3, pp.419-448, 2007.

[21] G. Y. Agboka, "Participatory Localization: A Social Justice Approach to Navigating Unenfranchised/Disenfranchised Cultural Sites," Tech. Commun. $Q$, vol. 22, no. 1, pp. 28-49, Jan. 2013.

[22] J. Bobeth, S. Schreitter, S. Schmehl, S. Deutsch, and M. Tscheligi, "User-Centered Design between Cultures: Designing for and with Immigrants," in Human-Computer Interaction - INTERACT 2013, vol. 8120, no. 65, P. Kotzé, G. Marsden, G. Lindgaard, J. Wesson, and M. Winckler, Eds. Berlin, Heidelberg: Springer Berlin Heidelberg, 2013, pp. 713-720.

[23] C. Spinuzzi, "The Methodology of Participatory Design,” Tech. Commun., vol. 52, pp. $1-12$, Apr. 2005

[24] D. Schuler and A. Namioka, Participatory Design: Principles and the Practices. Hillsdale, NJ: Lawrence Erlabum Associates, 1993.

[25] P. Ehn, "Scandinavian design: On participation and skill" in Participatory design: Principles and practices, D. Schuler \& A.i Namioka, eds. Hillsdale, NJ: Lawrence Erlabum Associates, 1993. pp. 41-77

[26] J. D. Gould and C. Lewis, "Designing for usability: key principles and what designers think," Communications of the ACM, vol. 28, no. 3, 1985.

[27] F. E. Ritter, G. D. Baxter, and E. F. Churchill, "User-Centered Systems Design: A Brief 
History," in Foundations for Designing User-Centered Systems, no. 2, London:

Springer, 2014, pp. 33-54.

[28] J. M. Carroll, "Encountering Others: Reciprocal Openings in Participatory Design and User-Centered Design," Human-Computer Interaction, vol. 11, no. 3, pp. 285-290, 1996.

[29] P. J. Larkin, B. Dierckx de Casterle, and P. Schotsmans, "Multilingual Translation Issues in Qualitative Research: Reflections on a Metaphorical Process," Qual. Health Research, vol. 17, no. 4, pp. 468-476, Apr. 2007.

[30] B. Temple and A. Young, "Qualitative Research and Translation Dilemmas," Qual. Research, vol. 4, no. 2, pp. 161-178, Aug. 2004.

[31] A. Voida, "Shapeshifters in the voluntary sector: exploring the human-centeredcomputing challenges of nonprofit organizations.," interactions, vol. 18, no. 6, pp. 2731, Nov - Dec, 2011.

[32] N. P. Moraras, "Implementation of the Affordable Care Act: Evaluation of Health Insurance Outreach and Enrollment Services ," International Community Health Services, Seattle, WA 2014.

[33] L. A. Goodman, "Snowball sampling," The annals of mathematical statistics, vol. 32, no. 1, pp. $148-170,1961$.

[34] J. Nielsen, "Why you only need to test with 5 users?," 19-Mar-2000. [Online]. Available: https://www.nngroup.com/articles/why-you-only-need-to-test-with-5-users/. 
[35] T. Gorman, E. Rose, J. Yaaqoubi, A. Bayor, and B. Kolko, “Adapting usability testing for oral, rural users,” in Proc. SIGCHI Conf. on Human Factors in Comp. Sytms., 2011, pp. 1437-1440.

[36] T. Boren and J. Ramey, "Thinking aloud: reconciling theory and practice," IEEE Trans. Profess. Commun., vol. 43, no. 3, pp. 261-278, 2000.

[37] M. Hughes, "Rigor in Usability Testing.," Tech. Commun. vol 46, no 4, pp. 488-94, 1999.

[38] A. K. Shenton, "Strategies for ensuring trustworthiness in qualitative research projects" Educ. for inform., vol. 22, no. 2, pp. 63-75, 2004.

[39] HRSA, The Affordable Care Act and Health Centers fact sheet. 2014.

[40] K. Pollitz, J. Tolbert, and R. Ma, "Survey of health insurance Marketplace assister programs,” Kaiser Family Foundation, Washington, 2014. [Online] Available: http://kff.org/health-reform/report/survey-of-health-insurance-marketplace-assisterprograms/

[41] Consumers Union, Measuring health insurance literacy: A call to action. A Report from the Health Insurance Literacy Expert Roundtable. 2012.

[42] M. Kutner, E. Greenburg, Y. Jin, \& C. Paulsen “The Health Literacy of America's Adults: Results From the 2003 National Assessment of Adult Literacy," National Center for Education Statistics pp. 1-76, Aug. 2006.

[43] S. Stern, "Knowledge Is Power: Focusing on Health Insurance Literacy May Increase 
Health Coverage Retention," Enroll America, 22-Aug-2014. [Online]. Available: https://www.enrollamerica.org/blog/2014/08/health-insurance-literacy-coverageretention/.

[44] J. Greene, E. Peters, C. K. Mertz, \& J. Hibbard "Comprehension and choice of a consumer-directed health plan: an experimental study.," The American J. of Managed Care, vol. 14, no 6, pp 369-367, 2008.

[45] L. J. Blumberg, S. K. Long, G. M. Kenney, and D. E. Goin, "Public understanding of basic health insurance concepts on the eve of health reform," Provider, vol 64, no 82, pp 52-54, 2013.

[46] R. Racadio, E. J. Rose, and B. E. Kolko, "Research at the margin: participatory design and community based participatory research,” in Proc. Participatory Design Conf, ACM, 2014, vol. 2, pp. 49-52

[47] E. J. Rose and J. Tenenberg, “Arguing about design: A taxonomy of rhetorical strategies deployed by user expereince practitioners," in Proc ACM SIG Design of Commun. 2016.

[48] L. Buley, The user experience team of one: a research and design survival guide. Brooklyn, NY: Rosenfeld Media, 2013.

[49] R. Buchanan, "Human dignity and human rights: Thoughts on the principles of humancentered design,” Design Issues, vol. 17, no. 3, pp. 35-39, 2001.

[51] J. Gothelf and J. Seiden, Lean UX: applying lean principles to improve user experience. 
Lean User Experience. Sebastopol, CA: O'Reilly Media, 2013.

[52] S. Krug, Rocket Surgery Made Easy: The Do-It-Yourself Guide to Finding and Fixing Usability Problems. San Francisco: New Riders Publishing, 2009.

[53] J. Nielsen. (1994) "Guerrilla HCI: Using Discount Usability Engineering to Penetrate the Intimidation Barrier" [Online] ,Available: https://www.nngroup.com/articles/guerrilla-hci/.

[54] D. Ralston, "The Positive Impact of Good UX on Non-Profits," User Exp. Mag., 11Feb-2014. [Online]. Available: https://uxmag.com/articles/the-positive-impact-of-goodux-on-non-profits.

Emma J. Rose is an Assistant Professor in the School of Interdisciplinary Arts \& Sciences at University of Washington, Tacoma, WA, USA. Her research is motivated by a commitment to social justice and a belief that the way technologies are designed ultimately shapes our world. Her research interests include the practice of user experience, how people use expertise to overcome resource constraints, and the development of technical identity. She teaches courses in technical communication, usability research methods, and user-centered design.

Robert Racadio is a senior design researcher. He uses qualitative and quantitative research methods to understand how to improve the healthcare experience for patients and healthcare providers. Robert has a Masters of Science and a PhD in Human Centered Design and Engineering from the University of Washington Seattle where he teaches courses in theory, usability, and user-centered design. 
Kalen Wong is an interaction and visual designer interested in visualizing data, designing enterprise applications, and using research to inform design. She has a Masters of Science in Human Centered Design \& Engineering from the University of Washington Seattle. She has worked in a variety of roles such as web design, program management, and user experience for Microsoft, T-Mobile and Google.

Shally Nguyen is a Business Analyst interested in improving bilingual material and bettering access to public technology for English-Limited immigrants. She holds a Bachelor of Arts in American Ethnic Studies from the University of Washington in Seattle.

Jee Kim is Curriculum Program Lead at SEIU 775 Benefits Group, the largest organization in the country dedicated to the development and education of Home Care Aides. The organization trains more than 45,000 home care aides a year in 16 languages. As the Outreach and Enrollment Coordinator at International Community Health Services, He led a team of in-person assisters implementing the Affordable Care Act in Washington State. He is an experienced community advocate and outreach specialist serving limited English proficient and underserved population.

Abbie Zahler is Assistant Director of Community Based Health at the Foundation for Healthy Generations and has worked in community health for more than nine years. She worked at International Community Health Services, a community health center serving immigrant and refugee communities in Seattle, where she led a team of community health workers and health educators to provide community and clinic based interventions to address health and social disparities. She has a wide range of experience working on immigrant and refugee health, project and program management, and partnership building. 